\title{
Manejo de la vía aérea en el perioperatorio de los pacientes infectados con COVID-19
}

\section{Perioperative airway management in patients infected with COVID-19}

Acad. Dr. Raúl Carrillo-Esper,* Dra. Leslían Mejía-Gómez, ${ }^{\ddagger}$

Dr. Jorge Sánchez-Rosendo, ${ }^{\S}$ Dr. Manuel Lomelí-Terán, ${ }^{\|}$Dr. Adrián Vázquez-Lesso, ${ }^{\llbracket}$

Dr. Augusto Pérez-Calatayud,** Dr. Ricardo Cabello-Aguilera ${ }^{\ddagger}$

RESUMEN. La enfermedad por coronavirus 2019 (COVID-19) es altamente contagiosa; su transmisión se efectúa predominantemente por propagación de gotas. De esta manera, los procedimientos durante el manejo inicial de la vía aérea y de las áreas críticas pueden generar gotas y aerosoles que incrementan el riesgo de transmisión. El propósito de este documento es realizar una recopilación reunida a corto plazo que pueda asesorar sobre el manejo de la vía aérea en pacientes infectados por COVID-19; este documento está diseñado para adaptarse de acuerdo con las políticas y guías locales.

ABSTRACT. Coronavirus disease 2019 (COVID-19) is highly contagious. Transmission is predominantly by droplet spread. Procedures during the initial handling of the airway and critical areas can generate drops and aerosols that increase the risk of transmission. The aim of this paper is to review the recommendations and guidelines related to airway management in patient infected by SARS-Cov-2.

\section{INTRODUCCIÓN}

A finales del mes de diciembre de 2019, se presentó en la ciudad de Wuhan, una provincia de Hubei en China, el brote de una neumonía viral que es consecuencia de un nuevo tipo de coronavirus $\mathrm{B}$, el cual fue denominado como COVID-19 (acrónimo del inglés coronavirus disease); posteriormente, de manera oficial, se declarará al virus como SARS-Cov-2. Este coronavirus, a la fecha, se ha convertido en una pandemia y en una emergencia de salud a nivel mundial según lo declarado por la Organización Mundial de la Salud(1).

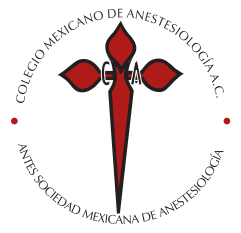

Palabras clave:

Coronavirus, COVID-19, vía aérea.

Keywords:

Coronavirus, COVID-19, airway.

* Academia Nacional de Medicina. División de Áreas Críticas del Instituto Nacional de Rehabilitación «Luis Guillermo Ibarra Ibarra».

‡ Servicio de Anestesiología. Unidad Médica de Alta Especialidad. Hospital de Traumatología y Ortopedia Lomas Verdes.

$\S$ Presidente del Colegio

Mexicano de Medicina Crítica.

" Jefe de la Unidad de Medicina Intensiva Hospital $\mathrm{H}+$, Querétaro.

" Director del Grupo

EMIVA Internacional.

** Jefe de División de Medicina Aguda. Hospital General de México.

¥ Director Médico del HMG

Hospital Coyoacán.

Solicitud de sobretiros:

Dr. Raúl Carrillo-Esper

Colegio Mexicano de

Anestesiología, A.C.

Revista Mexicana de Anestesiología. E-mail:

revistacmx@revistacomexane.com

Recibido para publicación: 23-03-2020

Aceptado para publicación: 09-04-2020

Se estima que el COVID-19 tiene una tasa de letalidad del $2 \%$, la cual es menor que la del síndrome respiratorio agudo grave (SARS, por sus siglas en ingles) (10\%) y que la del síndrome respiratorio de oriente medio (MERS, por sus siglas en inglés), pero es significativamente más alta que el virus de la influenza H1N1 $(0.026 \%)$.

No se tiene certeza aún del período de incubación, pero, según los datos de la OMS, es de dos a 10 días en promedio. De allí, las presentaciones típicas serán de cuatro a siete días antes del inicio de los síntomas. Se tiene reportado un caso que sugiere que la transmisión puede ocurrir durante el período 
asintomático, lo que podría representar desafíos al momento del diagnóstico y control de estos pacientes.

La presentación clínica más frecuente es fiebre, tos y disnea, y en un $17 \%$ de los pacientes no presenta ningún cuadro febril inicial. Los estudios de imagen muestran neumonía bilateral con un importante compromiso intersticial y subpleural. Un buen número de pacientes presentan una forma leve de la enfermedad, pero hasta un $6 \%$ presentan la forma grave, que se caracteriza por una lesión pulmonar aguda que requiere de intubación orotraqueal y ventilación mecánica; de este grupo de pacientes, un elevado porcentaje fallece.

El manejo de la vía aérea en los pacientes con la forma grave es clave para su recuperación. El problema es que este procedimiento ha sido catalogado como de muy alto riesgo de contagio para el personal de salud. De esta manera, se han publicado diferentes guías de práctica para optimizar la intubación orotraqueal en el sentido de optimizar la seguridad de este procedimiento y disminuir el riesgo de contagio.

Por esta razón, el objetivo de este trabajo es presentar a los interesados una serie de recomendaciones elaboradas con base en la evidencia científica y en la experiencia de varios grupos de trabajo que se relaciona con el manejo de la vía aérea en el perioperatorio de los pacientes infectados por el virus SARS-Cov-2 ${ }^{(2)}$.

\section{1) Infección por virus SARS-Cov-2}

El SARS-Cov-2 es un virus corona encapsulado de ácido ribonucleico (ARN) monocatenario altamente contagioso. La transmisión ocurre predominantemente por propagación de gotas y aerosoles, además de por contacto directo con el paciente o fómites (superficies plásticas, vidrio, madera, papel, acero); algunas de las partículas más pequeñas de este virus pueden permanecer en el aire por algún tiempo.

Los procedimientos durante el manejo inicial de la vía aérea y en la Unidad de Cuidados Intensivos (UCI) pueden generar aerosoles que incrementan el riesgo de transmisión ${ }^{(2,3)}$. El personal de salud que debe tratar a pacientes con infección por COVID-19 tiene un mayor riesgo de contraer la enfermedad (5\% de riesgo de contagio con el equipo de protección personal [EPP] $)^{(4)}$, debido a que la mayor carga viral de SARS-CoV-2 aparece en las secreciones. La intubación traqueal es un procedimiento potencialmente de alto riesgo para el que la provee a la vía aérea, particularmente porque se arriesga a la exposición de una carga viral alta y porque la transmisión es directa, además de que ello se asocia con una enfermedad más grave.

Por esta razón, se deben tomar todas las precauciones necesarias. Una de ellas, es la protección del personal que reduzca la exposición viral. En la actualidad, existe un amplio asesoramiento que se actualiza periódicamente sobre la prevención y el control de infecciones relacionadas con COVID-19.

Como se ha mencionado, el SARS-CoV-2 se transmite por inhalación directa de partículas que pueden viajar hasta dos metros o por exposición de superficies contaminadas (fómites). Los procedimientos generadores de aerosoles crean un mayor riesgo de transmisión de infección. Una revisión sistemática de 2012 del riesgo de infección para los trabajadores de la salud ${ }^{(5)}$, la cual estuvo basada en la literatura, clasificó los procedimientos de las vías respiratorias en orden descendente de riesgo, tal como la intubación traqueal y la traqueotomía. El uso de dispositivos de alto flujo de oxígeno, ventilación no invasiva y fibrobroncoscopía no están recomendados, a causa de los aerosoles que generan; esto incrementa el riesgo de transmisión y contagio. Otros procedimientos potencialmente generadores de aerosoles incluyen la desconexión de los circuitos de ventilación y los procedimientos de aspiración, entre otros ${ }^{(4)}$.

\section{2) Preparación para la intubación endotraqueal en pacientes infectados con SARS-Cov-2}

Los anestesiólogos y demás equipo médico (intensivistas y urgenciólogos), que manejarán a pacientes graves que han sido infectados por SARS-Cov-2 y que presentan la forma grave de esta infección, estarán en la primera línea de manejo, ya que estos pacientes requerirán el abordaje avanzado de la vía aérea por personal experto en su manejo. Luego de aprender de experiencias previas con SARS y MERS junto con el conocimiento de los factores epidemiológicos actuales del COVID-19(4), es de importancia extrema tener el conocimiento de prevención y control de infecciones, vigilancia en medidas de protección, estricta adhesión a la colocación y retiro del equipo de protección y la preparación para el cuidado de pacientes infectados. Asimismo, el riesgo de insuficiencia respiratoria en este tipo de pacientes es considerable y requiere el apoyo de un equipo transdisciplinario para su atención.

La intubación traqueal del paciente con COVID-19 es un procedimiento de alto riesgo para el personal, independientemente de la gravedad clínica de la enfermedad. Por ello, debemos tener en cuenta estos tres rubros:

- Seguro para el personal y el paciente.

- Preciso para evitar técnicas poco confiables, desconocidas o repetidas.

- Rápido, que nos permita ser oportuno, sin prisas ni demoras.

\section{3) Protección del personal: prioridad en pacientes con coronavirus}

En la Tabla 1 se resume el equipo de protección personal o de aislamiento de sustancias corporales (ASC) para el personal de 
Tabla 1: Equipo de protección personal o de aislamiento de sustancias corporales para el personal de salud.

1. La higiene de manos es fundamental

2. Protección ocular de montura integral o protector facial completo

3. Mascarilla de alta eficacia (FFP2 o FFP3) o mascarilla facial n-95 (partículas de 3 micras)

4. Es importante resaltar que la mascarilla de careta no ofrece protección para las partículas en aerosol del virus

5. Batas resistentes o impermeables a fluidos. Protección ocular con extensión lateral, careta completa, y filtros o cubrebocas tipo N95; también se deben utilizar cubiertas para el cabello o capuchas (trajes completos de aislamiento). Se recomienda utilizar guantes de manga larga. Se puede utilizar cinta colocada de manera vertical para mantener los guantes adheridos a la bata (no utilizarlos de manera circunferencial ya que esto hace más difícil la manipulación y la eliminación de estos insumos)

6. La protección del calzado puede aumentar el riesgo de autocontaminación, por lo que debe evitarse de manera inicial

7. El calzado debe ser impermeable y poder ser susceptible a descontaminación

8. De preferencia, los overoles con capucha deben ser usados como capa interior debajo del equipo de protección personal

9. Utilizar doble guante

10. Si está disponible, una alternativa es utilizar sistemas de respiradores purificadores de aire para cuando se realicen los procedimientos con generación de aerosoles como la intubación endotraqueal (RPAP)

11. Sin dispositivos más costosos y con mayor factor de protección que el N95, sin embargo, deben estar disponibles en caso de que se requiera una intervención más prolongada con el paciente

12. Se ha documentado la infección del personal de salud aun utilizando N95, sobre todo en situaciones prolongadas, por lo que en escenarios de alto riesgo se prefieren los sistemas RPAP

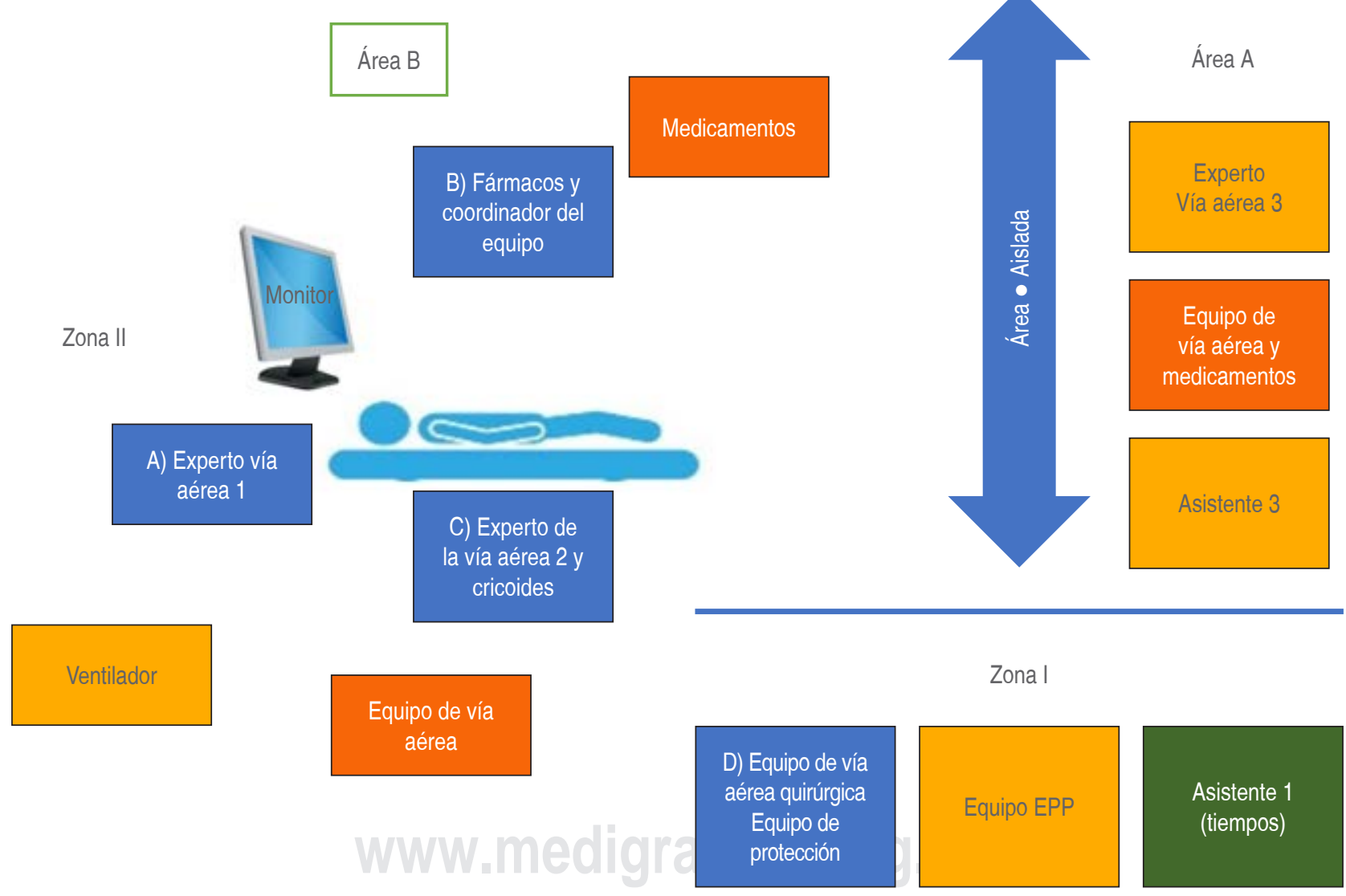

Figura 1: Manejo de la vía aérea. Los roles del equipo de las tres personas expertas en el manejo de la vía aérea y asignadas para el manejo de COVID-19 serán: A) experto vía aérea 1 (mayor experiencia y manejo de la vía aérea). B) Administrador de fármacos y líder del equipo. C) Experto de la vía aérea 2 y aplicador de presión cricoides. D) El equipo de expertos de la vía quirúrgica deben estar alertas al llamado con equipos de protección y deben estar fuera del área aislada para evitar contagios. Estos roles pueden cambiar después del primer intento fallido de intubación, momento en el que el segundo intubador se activa. Si el equipo consta sólo de un experto de vía aérea, los roles permanecen sin cambios entre los intentos de intubación. Recurra de inmediato a la ayuda de expertos en la vía aérea quirúrgica. En la zona 1, se encuentran los asistentes 2 y 3; se debe tomar tiempos para apoyar ambas zonas. 
$\operatorname{salud}^{(6,7)}$. Es importante tener asignada el área de aislamiento, que sea exclusivamente para paciente con sospecha o de caso confirmado de infección con COVID-19. Todos deben conocer el plan de verificación, y para lograrlo se debe hacer previamente una buena estrategia de planeación. Estos espacios deben estar conformadas por las siguientes dos áreas A y B:

A) El área de atención donde se encuentra todo el equipo necesario para el manejo del paciente que evite la necesidad de tránsito del equipo, insumos y personal a esta zona, y una antesala con suficiente espacio donde el equipo de salud pueda colocarse y retirarse el equipo de aislamiento de sustancias corporales.

B) El área de aislamiento se debe dividir en dos zonas: 1 y 2. La zona 1 debe cumplir los requerimientos de presión negativa, de intercambio (12 cambios por minuto de aire frecuente) y filtros hidrofóbicos de purificación del mismo $^{(8)}$. El área debe contar con un monitor para la evaluación hemodinámica, de capnografía, así como con ventilador mecánico, máquina de anestesia, una camilla y un carro de vía aérea de acceso difícil exclusivamente para ser usado en pacientes con COVID-19; también debe haber medicamentos de choque.

Se debe limitar el personal presente durante la intubación traqueal $^{(9)}$. Se propone un experto de la vía aérea y un segundo experto para auxiliar, así como un tercero como coordinador y líder del equipo que administre los medicamentos y anote los tiempos (medicamentos y horarios).

Para limitar la zona 1 debe existir una segunda área o zona 2, la cual debe estar conformada por equipos e insumos de ayuda inmediata, que sirvan para el manejo de la vía aérea y medicamentos como un carro rojo. Se debe disponer de un equipo entrenado exclusivamente para acceder al área quirúrgica, que atienda únicamente a los pacientes infectados por COVID-19 y que se encuentre en la zona 2; ello para limitar al personal y la exposición a la contaminación. Este equipo debe estar siempre alerta al llamado del personal que se encuentre en la zona 1 . Su distribución se realizará como se muestra en la Figura 1.

En el caso del manejo de la vía aérea de acceso difícil y estando presente una emergencia de «no intubo, no ventilo», las recomendaciones de equipamiento necesarias para el equipo de vía aérea de acceso difícil se resumen en la Tabla 2.

Las recomendaciones actuales refieren que no se deben utilizar mascarillas laríngeas, debido a la gran diseminación viral que pueden tener, además de no utilizar aerosoles para la intubación, debido al riesgo de mayor diseminación del virus.

A continuación, se describen algunos puntos de ayuda cognitiva:

1. Trabajo en equipo con el líder, asignación de roles $\mathbf{y}$ colaboración efectiva (Figura 1).
2. El carro especial de la vía aérea asignado para COVID-19 debe estar a la cabecera del paciente (Tabla 2).

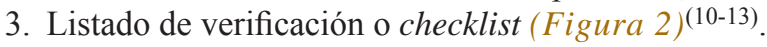

4. Algoritmo de actuación para el abordaje de la vía áerea (Figuras 3 y 4$)^{(11-13)}$.

\section{4) Lista de verificación y capacitación del factor humano para el manejo de la vía aérea en el paciente con COVID-19}

La lista de verificación de intubación traqueal, diseñada y modificada para ayudar a la preparación debe verificarse antes de ingresar a la habitación del paciente infectado con COVID-19. Es importante realizar la capacitación mediante simulación del personal médico experto en el manejo de la vía aérea, que permita realizar el abordaje en pacientes con COVID-19 para un mejor manejo y control, y para evitar fallos. El fracaso en el manejo de la vía aérea está en el factor humano: la desorganización del equipo, la falta de entrenamiento en el manejo de la vía aérea, las influencias ambientales y el desempeño individual $^{(10,11)}$.

La técnica recomendada para el abordaje e instrumentación de la vía aérea en estos escenarios es aquélla que cumple con los siguientes objetivos en orden prioritario:

1. La seguridad del equipo de salud.

2. El aislamiento de manera efectiva del paciente infectado.

3. El establecimiento de las técnicas más efectivas al momento de abordar e instrumentar la vía aérea.

4. La resolución de la insuficiencia respiratoria.

5. El evitar el contagio a los demás miembros del equipo de salud y a los pacientes.

Es importante centrarse en la seguridad, la rapidez y la fiabilidad, y en el éxito del primer intento de la intubación, ya que los múltiples intentos incrementan el riesgo de diseminación en el personal de salud ${ }^{(12)}$.

Tabla 2: Carro de vía aérea equipado exclusivamente para el uso en pacientes infectados con COVID-19.

1. Laringoscopios desechables

2. En caso de no contar desechables, se deben utilizar laringoscopios con hojas intercambiables de todos los números (se recomienda después de su uso, colocar en una bolsa estéril y enviar a esterilización)

3. Bougies desechables 0 esterilizar con medidas estrictas

4. Guías para intubación

5. Sondas orotraqueales

6. Cánulas de Guedel

7. Videolaringoscopios con palas intercambiables

8. Fibrolaringoscopio

9. Equipo de vía aérea quirúrgica 


\section{Fuera de área}

\section{Checklist del PPE: \\ * Sé minucioso, cauteloso \\ y no te apresures \\ * Lavarse las manos, \\ * Ponerse el PPE, con las medidas de protección \\ * Mascarilla FFP3 \\ * Guantes \\ * Protector de pelo \\ ${ }^{*}$ Botas desechables \\ * El equipo de protección debe ser desechable \\ * Verificar el equipo \\ asignado para el abordaje \\ de la vía aérea \\ * Identificación del nom- \\ bre del personal en las \\ vísceras o lentes}

\section{Asignar roles:}

1. Líder del equipo (medicamentos, tiempos)

2. Primer intubador

3. Segundo intubador y presión crinoidea

*¿Quién lleva los tiempos?

\section{${ }^{*}$ Checklist:}

* Fuente de oxígeno

* Máquina de anestesia

* Medicamentos

${ }^{*}$ Monitor

*Ventilador

* Tiempo fuera

* Tener listo equipo de eFONA (front of neck access, emergency) "Acceso quirúrgico de emergencia"

Contactar al personal requerido para ayudar en la atención

Preparación del
material:
Monitoreo:
SatO
EtCo
ECG
T/A
* Circuito Mapleson C
* Circuito anestésico
* Intercambiador de calor
y humedad (HME)
" Evitar mascarilla -bolsa-
reservorio
* Guedel
* Succión
*Videolaringoscopía
* Bougie
* Estilete
* Dos tubos endotra-
queales
* Tiras y jeringas (neumo-
taponamiento)
* Dispositivos supraglóti-
cos (en caso de urgencia)
no recomendado
* Equipo quirúrgico
disponible. eFONA
Medicamentos
Tener disponible:
* Bloqueadores
Neuromusculares
* Inductores
Considerar:
(ketamina)
* Inductores
*Vasopresores
* Inotrópicos
* Mantenimiento de la
sedación

\section{¿¿La vía aérea es difícil? \\ * ¿Podemos despertar al paciente si la intubación falla? \\ * Considerar ISR \\ Intubación de secuencia rápida \\ * ¿Cuál es el plan para una vía aérea difícil? \\ - Plan A: fármacos \\ Y laringoscopía \\ Videolaringoscopio \\ Recomendado (des- echable)}

- Plan B/C:

Dos manos

Dispositivo supraglótico

- Plan C:

Equipos quirúrgicos eFONA

\section{Bougie- guías-estiletes}

* ¿Alguien tiene una duda 0 inquietud?

\section{En el área}

Preparación de

paciente:

* Lavado de manos (personal en sanitarios) PPE y toda la protección

${ }^{*}$ Accesos venosos

Optimizar posición

* Posición de rampa

* Considerar posición de

Trendelenburg

Evaluación de la vía aérea

* Valoración de vía aérea

* Intubación secuencia rápida (ISR)

* Localizar membrana cricotiroidea.

Preoxigenación adecuada

* 3 minutos o $\mathrm{EtO}_{2}>85 \%$

* Considerar:

- Flujos bajos

- No usar mascarilla bolsa-reservorio

En caso de no contar con el equipo adecuado usar mascarilla facial con buen sellado bimanual para evitar diseminación viral - Ayuda del segundo intubador para ventilación Optimizar condiciones del paciente:

* Vasopresor/inotrópicos

* Optimización de líquidos

* Peso

${ }^{*}$ Alergias

* Riesgo de hipercalemia

Evitar succinilcolina

\section{Posterior}

Manejo de la vía aérea:

${ }^{*}$ Conectar al ventilador

*Checar capnógrafo

* Asegurar SOT

* Evite desconexiones innecesarias

Otras

* Colocar sonda nasogástrica

* Colocar sonda urinaria

* Tomar cultivo traqueal.

*Eliminación cuidadosa del equipo

* Descontaminación inmediata del material reusable

* Retirar adecuadamente el EPP

- Ser extremadamente meticuloso

- Lavado adecuado de manos

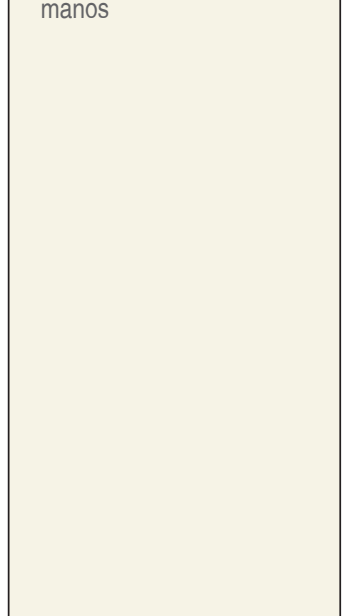

Figura 2: Listado de verificación o checklist de intubación. COVID-19 para intubación traqueal de emergencia.

Tomado de: Apfelbaum JL, Hagberg CA, Caplan RA, Blitt CD, Connis RT, Nickinovich DG, et al. Practice guidelines for management of the difficult airway: an updated report by the American Society of Anesthesiologists Task Force on Management of the Difficult Airway. Anesthesiology. 2013;118:251e70. Adaptado y modificado para COVID-19 por Carrillo-Esper, Mejía-Gómez. 2020. 
Lista de verificación: equipo de protección para el personal de salud y plan estratégico para el manejo de la vía aérea. Experto en el manejo de la vía aérea

\section{$\checkmark$}

Preoxigenación y checklist

1. Monitoreo

2. Posición de la cabeza (rampa si es posible)

3. Evaluación de la vía aérea (clínica, ultrasonido)

4. Identificar la membrana cricotiroidea (en caso de fallo en la intubación)

5. Capnografía

6. Preoxigenación. Puntas de alto flujo, evitar sistema bolsa-mascarilla-reservorio. Para mantener $\mathrm{FiO}_{2}$ 90-97\% (lo más cercano a 100\%)

7. Circuito anestésico/Mapleson C

8. Intercambiador de calor y humedad (HME)

9. Filtros HEPA (high) aire

\section{$\downarrow$}

Plan «A»

Laringoscopía intubación traqueal

1. Se recomienda al primer intento (máximo de tres intentos)

2. Rocuronio $1.2 \mathrm{mg} / \mathrm{kg}$, succinilcolina $1.5 \mathrm{mg} / \mathrm{kg}$

3. Evitar el uso de aerosoles como la lidocaína en espray (diseminación viral)

4. Presión cricotiroidea

5. Uso de laringoscopía directa (laringoscopio desechable) recomendación: videolaringoscopio con palas desechables + bougie 0 estiletes desechables

6. Presión cricoidea
Anotar tiempos

Checar inserción adecuada de la sonda orotraqueal y el capnógrafo

- Ultrasonido

- Estetoscopio

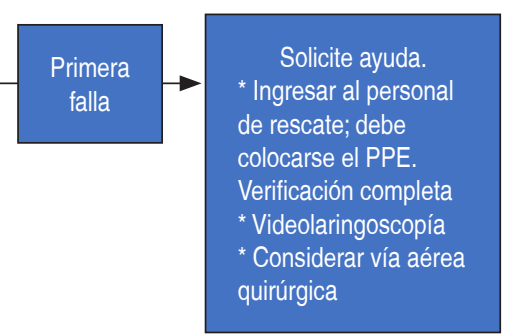

Plan «B/C» oxigenación de rescate

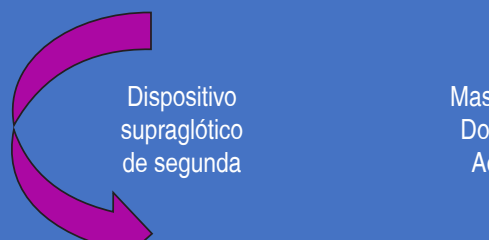

Mascarilla facia

Dos personas

Adyuvantes

Máximo tres intentos Cambiar de dispositivo operador Abrir eFONA

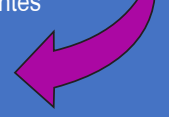

Éxito

Solicite ayuda

* Despertar al

paciente

${ }^{*}$ Dispositivo supraglóti-

co de rescate

* eFONA

\section{Figura 3:}

Algoritmo de actuación en el abordaje de la vía aérea difícil de urgencia en el paciente con COVID-19. Tomado de: Gómez-Ríos MA, Gaitini L, Matter I, Somri M. Guidelines and algorithms for managing the difficult airway. Rev Esp Anestesiol Reanim. 2018; 65: 41. Modificado y adaptado para COVID-19 por Carrillo-Esper, Mejía-Gómez. 
- Extender cuello

- Bloqueo neuromuscular

- Continuar con ventilación de rescate

- Excluir el fallo del oxígeno o circuito anestésico

- Usar eFONA

- Considerar cricotiroidotomía con bisturí

Checklist en el equipo de rescate de la vía aérea y la protección para el personal de salud: plan estratégico para el manejo de la vía aérea y proveedor más experimentado en vía aérea quirúrgica

\section{(FONA)}

Cricotiroidectomía con bisturí

Equipo:

1. Hoja ancha número 10020

2. Bougie $(<4 \mathrm{fr})$

3. Tubo (5-6 diámetro interno con balón)

* Palpación laríngea para identificar membrana cricotiroidea

Membrana cricotiroidea palpable

Incisión transversal a través de la membrana cricotiroidea

Girar la hoja 90 grados (borde afilado a los pies)

Deslizar la punta del bougie a través del corte al interior de la tráquea

Presionar el tubo lubricado hacia al bougie y hacia la tráquea

Inflar el balón y confirmar la posición con la capnografía

Fijar el tubo

Membrana cricotiroidea no palpable

Realizar una incisión vertical en la línea media

Disección para separar los tejidos

Identificar y fijar la tráquea

Proceder con la técnica de membrana cricotiroidea, palpable indicada arriba

Figura 4:

Algoritmo de actuación abordaje de la vía aérea difícil de urgencia, escenario «NO puedo ventilar NO puedo intubar», modificado a paciente con COVID-19.

Tomado de: Gómez-Ríos MA, Gaitini L, Matter I, Somri M. Guidelines and algorithms for managing the difficult airway. Rev Esp Anestesiol Reanim. 2018;65:41-48. Modificado y adaptado al español por Carrillo-Esper, Mejía-Gómez. 
Consideraciones previas a la intubación:

1. Lo más segura y efectiva posible.

2. Menor exposición con líquidos corporales del paciente, en especial gotas $\mathrm{y} / \mathrm{o}$ aerosoles.

3. Menor diseminación al área de trabajo de las secreciones del paciente.

4. Lo más rápido posible en tiempo, con un mínimo de complicaciones.

5. Los instrumentos deben ser desechables; en caso de no serlo deben ser lo más aislado posibles de contacto con el paciente.

6. Se debe hacer énfasis que los portadores deben ser lo más expertos posibles para garantizar el mínimo de complicaciones.

7. Disminuir al máximo el personal requerido para la atención del paciente.

\section{5) Abordaje de la vía aérea}

En la Tabla 3 se resumen las recomendaciones para el abordaje de la vía aérea en pacientes infectados con COVID-19 ${ }^{(14,15)}$.

\section{Previamente al manejo de la vía aérea:}

- Trate a cada paciente como potencial propagador de COVID-19.

- Protégete a ti mismo y a los demás.

- El personal que esté en contacto con el paciente durante el manejo de la vía aérea y la intubación deberá de seguir de manera puntual y estricta las instrucciones para el uso y colocación del equipo de protección personal (EPP).

- Suspenda la cirugía electiva en pacientes con COVID-19 (esto incluye pacientes con tos o síntomas de resfriado sin fiebre).

- Evalúe exhaustivamente la vía aérea para evitar el uso de múltiples dispositivos de esta misma vía y exposición $\operatorname{prolongada}^{(15)}$.

- Lave exhaustivamente sus manos antes o después de estar con el paciente o luego de realizar cualquier procedimiento.

- Tenga listo el carro de vía aérea y/o todos los dispositivos necesarios para el manejo de la misma; en éste se incluyen videolaringoscopios $^{(16)}$.

- Se recomienda el uso de equipo desechable para el manejo de la vía aérea (en caso de contar con el éste).

- Reúna a todo el equipo de trabajo para planificar el abordaje de la vía aérea. En lo posible desarrolle un protocolo de atención relacionado con el mismo.

- En lo posible, organice un equipo de manejo de vía aérea COVID-19.

- Refuerce las prácticas estrictas de control de infecciones; esto incluye recordar que el equipo integral de limpieza tenga asignados con trajes EPP especiales.
- Tener listo el equipo de eFONA (Front of neck access, emergency) «Acceso quirúrgico de emergencia».

- Limpie continuamente y con base a la normatividad las superficies de trabajo, la máquina de anestesia y otros fómites.

\section{Durante el manejo de la vía aérea:}

- Se recomienda, acorde con los recursos hospitalarios, el uso de filtros HEPA. Este tipo de filtro es capaz de atrapar el $99.5 \%$ de partículas de más de 0.003 micras de diámetro en una habitación de 50 metros cuadrados y con un flujo de aire de 190 metros cúbicos por hora.

- La técnica elegida puede diferir de acuerdo con las prácticas y equipos locales.

- Realice la preoxigenación con una máscara bien ajustada y con un circuito Mapleson C o circuito anestésico. Evite en todo momento la generación de aerosoles. Se puede usar la técnica de dos manos con aislamiento de la cara del paciente y con un asistente que ventile con la bolsa reservorio. La ventilación con mascarilla deberá realizarse a baja presión y flujos bajos de oxígeno, así como con un sistema cerrado. En caso de que el paciente mantenga una adecuada saturación de la hemoglobina, la preoxigenación se puede llevar a cabo con el flujo de oxígeno por puntas nasales que no rebase los seis litros/minuto.

- No utilice presión positiva al momento de la preoxigenación, pues genera gran cantidad de aerosol.

- No utilice altos flujos de oxígeno > 6 litros, ya que genera aerosoles.

- No utilice ventilación no invasiva, al menos que se tengan los filtros y aditamentos adecuados y que el paciente se encuentre en un cuarto de presión negativa que haga 12 recambios de aire por hora.

- Es importante evitar los aerosoles al momento de la intubación, extubación y durante la aspiración de secreciones.

- De preferencia la aspiración de secreciones debe realizarse por sistema cerrado.

- No se recomienda traqueostomía, salvo en situaciones emergentes.

- No use mascarilla bolsa-reservorio.

- Se recomienda el uso de laringoscopio desechable. En caso de no disponer de este dispositivo deberá de usar un laringoscopio tradicional, el cual después de utilizarlo deberá depositarse en una bolsa plástica sellada y enviarse a esterilizar en óxido de etileno a temperatura no mayor a 60 grados centígrados.

- Se recomienda videolaringoscopía con palas desechables para intubación traqueal.

- En caso de emergencia, emplee un dispositivo de la vía aérea supraglótica de segunda generación para el rescate de la vía aérea. 
Tabla 3: Recomendaciones para el abordaje de la vía aérea en pacientes infectados con COVID-19.

Antes

Trate a cada paciente como potencial propagador de COVID-19

Protégete a ti mismo y a los demás

No hay operación electiva para pacientes con síntomas de COVID-19 (esto incluye pacientes con tos o síntomas de resfriado y sin fiebre)

Excluya al paciente que ha visitado recientemente áreas de alto riesgo. Esta es una situación dinámica, así que manténgase actualizado

Evalúe exhaustivamente la vía aérea para evitar el uso de múltiples dispositivos de esta vía y evite la exposición prolongada

Refuerce las prácticas estrictas de control de infecciones, esto incluye recordar que el equipo integral de limpieza tenga asignados

trajes PPE especiales y personalizados para que limpie las superficies de trabajo, la máquina de anestesia y otros fómites

Considere usar una máscara quirúrgica durante el manejo de la vía aérea

Use guantes dobles. Recuerde que el uso inapropiado de guantes puede ser contraproducente en la prevención de infecciones

Quítese inmediatamente el guante superior del guante después de la inserción de la vía aérea, antes de tocar cualquier otro objeto, incluido el circuito de respiración y la bolsa del depósito

Durante

Utilice técnicas confiables que funcionen incluso cuando se encuentre con dificultades

No se deben usar nebulizaciones

Considere la intubación de secuencia rápida y la presión cricoidea donde un asistente capacitado pueda aplicarlo; quítelo si causa complicación a la visualización

En la habitación debe utilizarse un filtro HEPA ${ }^{\circledR}$ (high efficiency particulate air)

El filtro es capaz de atrapar el $99.5 \%$ de partículas de más de 0.003 micras de diámetro en una habitación de 50 metros cuadrados y con un flujo de aire de 190 metros cúbicos por hora

La técnica elegida puede diferir de acuerdo con las prácticas y equipos locales

La preoxigenación se realizará con una máscara bien ajustada y un Mapleson $\mathrm{C}$ o circuito anestésico

Durante 3-5 minutos, o $\mathrm{EtO}_{2}>85 \%$

No utilice la presión positiva al momento de la preoxigenación, pues genera gran cantidad de aerosol

No utilice altos flujos $>6$ litros para evitar diseminación viral

No utilice ventilación no invasiva al menos que se tengan los filtros y aditamentos adecuados y que el paciente se encuentre en un

cuarto de presión negativa que haga 12 recambios de aire por hora

Es importante evitar los aerosoles al momento de la intubación, la extubación y durante la aspiración, debido a la presión negativa que se extrae (< de $30-40 \mathrm{~cm}$ de secreciones)

Se recomienda la ventilación de alta frecuencia. Las máquinas de anestesia no cuentan con filtros de alta eficiencia hidrofóbicos

No se recomienda traqueotomía, salvo en situaciones emergentes

No use mascarilla bolsa-reservorio

Se recomienda laringoscopio desechable en caso de contar con ello; se debe utilizar una bolsa estéril después de su utilización para una estricta esterilización

Se recomienda videolaringoscopía con palas desechables para intubación traqueal

Evite la ventilación con mascarilla a menos que sea necesario y use una técnica de baja presión y bajo flujo para dos personas si es necesario

La ventilación con máscara para dos personas y dos manos mejora el sellado

En caso de emergencia, emplee el dispositivo de la vía aérea supraglótica de segunda generación para el rescate de la vía aérea

No se recomiendan el uso de dispositivos supraglóticos

Coloque un filtro HME (intercambiador de calor y humedad) entre el montaje de la sonda orotraqueal y el circuito en todo momento Se debe evitar el uso de oxígeno nasal de alto flujo, ventilación no invasiva, broncoscopía y succión traqueal, a menos que exista un sistema de succión en línea

Monitoreo completo

Para evitar el colapso cardiovascular, se recomienda ketamina 1-2 mg/kg-L, lidocaína 1-1.5\% vía intravenosa (disminuye el 30\%)

Para el bloqueo neuromuscular use rocuronio $1.2 \mathrm{mg} / \mathrm{kg}$ o succinilcolina $1.5 \mathrm{mg} / \mathrm{kg}-\mathrm{L}$

El vasopresor para bolo o infusión debe estar disponible de inmediato para controlar la hipotensión

Confirme la posición; es difícil usar EPP

Infle el manguito traqueal para sellar las vías respiratorias antes de comenzar la ventilación

Confirme la intubación traqueal con capnografía, ultrasonido o estetoscopio

Utilice un algoritmo de intubación traqueal fallido estándar con una ayuda cognitiva si surge alguna dificultad

Comuníquese claramente: instrucciones simples, comunicación de circuito cerrado (repita las instrucciones) con un volumen adecuado, sin gritos

Coloque una sonda nasogástrica después de que se complete la intubación y se establezca la ventilación de manera segura

Coloque inmediatamente el bougie en un contenedor amarillo después de usarlo

Si COVID-19 aún no está confirmado, tome un aspirado traqueal profundo para panel viral y use succión cerrada

Deshágase del equipo desechable de manera segura después de su uso

Descontamine el equipo reutilizable por completo de acuerdo con las instrucciones

Después de salir de la habitación, asegúrese de que la eliminación del EPP sea meticulosa

Sala limpia 20 minutos después de la intubación traqueal (o último procedimiento de generación de aerosol)

Un registro visual de la intubación traqueal debe ser visible en la habitación del paciente

Si hubo dificultad al momento de la intubación, el plan de vía aérea difícil debe mostrarse en la habitación y comunicarse entre turnos 
Continúa Tabla 3: Recomendaciones para el abordaje de la vía aérea en pacientes infectados con COVID-19. T

\begin{tabular}{ll} 
Después & Conecte el circuito del ventilador mecánico lo antes posible \\
& Durante la transferencia, coloque el ventilador en modo de espera \\
& $\begin{array}{l}\text { Retírese la segunda capa de guantes después de transferir el paciente al ventilador } \\
\text { Lávese las manos o use gel para manos } \\
\text { Evite estrictamente tocarse la cara, la nariz, los ojos y el cabello }\end{array}$ \\
\hline $\begin{array}{l}\text { Durante la } \\
\text { extubación }\end{array}$ & $\begin{array}{l}\text { Considere la extubación en un cuarto especial para minimizar la exposición } \\
\text { Tome las mismas precauciones que durante la intubación (PPE, guantes, mascarilla, etcétera) } \\
\text { Deseche inmediatamente el dispositivo de vía aérea en el contenedor después de su uso }\end{array}$
\end{tabular}

- No se recomiendan el uso de dispositivos supraglóticos.

- Coloque un filtro HME (intercambiador de calor y humedad) entre el montaje de la sonda orotraqueal y el circuito en todo momento.

- Para evitar el colapso cardiovascular, se recomienda emplear ketamina $1-2 \mathrm{mg} / \mathrm{kg}-\mathrm{L}$, lidocaína $1-1.5 \%$ de forma intravenosa (disminuye el 30\%)

- Para el bloqueo neuromuscular use rocuronio $1.2 \mathrm{mg} / \mathrm{kg}$ o succinilcolina $1.5 \mathrm{mg} / \mathrm{kg}$ (que no tenga datos de hipercalemia).

- El vasopresor/inotrópico debe estar de acuerdo con el estado hemodinámico de cada paciente.

- Calibre el neumotaponamiento con manómetro de la sonda orotraqueal para asegurar las vías respiratorias antes de comenzar la ventilación y, de esta manera en lo posible, evite la generación de aerosoles.

- Confirme la intubación traqueal con capnografía y ultrasonido. Evite en lo posible el uso de estetoscopio, y si lo utiliza asegurar su estricta limpieza y esterilización.

- En caso de vía aérea fallida y difícil, se recomienda utilizar el algoritmo recomendado en la Figura 2.

- Comuníquese claramente: instrucciones simples, comunicación de circuito cerrado (repita las instrucciones) con un volumen adecuado y sin gritos.

- Coloque una sonda nasogástrica después de que se complete la intubación y se establezca la ventilación de manera segura.

- Se recomienda el uso de filtros HEPA en la unión del tubo endotraqueal con el circuito de la máquina de anestesia y/o ventilador mecánico, así como la rama espiratoria del circuito.

\section{Posterior al manejo de la vía aérea:}

- La ropa y el material desechable utilizados durante el procedimiento deberán ser depositados en bolsas y en contenedores de material biológico infecto-contagioso para su tratamiento y eliminación.

- Se deberá seguir estrictamente el proceso del retiro del equipo de protección personal.

- Descontamine el equipo reutilizable por completo acorde con la normatividad.
- Después de salir de la habitación, asegúrese de que la eliminación del EPP sea meticulosa.

- La sala debe estar limpia 20 minutos después de la intubación traqueal (o último procedimiento que pudo haber generado aerosoles).

- Si hubo dificultad al momento de la intubación, el plan de la vía aérea difícil debe de notificarse y comunicarse a todo el equipo.

\section{Durante la extubación:}

- Considere la extubación en un cuarto especial para minimizar la exposición.

- Tome las mismas precauciones que durante la intubación (EPP, guantes, mascarilla, etcétera).

- Deseche inmediatamente el dispositivo de vía aérea en el contenedor después de su uso.

\section{Videolaringoscopía}

Deben utilizarse videolaringoscopios para garantizar la efectividad del procedimiento, según las recomendaciones para el abordaje de la vía aérea. Algunas referencias se inclinan al tipo de videolaringoscopios con pantalla desacoplada a la pala o videolaringoscopio con pantalla acoplada, también de pala con canal para tubo o sin canal para tubo endotraqueal.

a) Técnica para la intubación endotraqueal con videolaringoscopio con hoja con canal

Ducanto $^{(13-15)}$ describe una técnica con videolaringoscopía para realizar la intubación endotraqueal en pacientes con COVID-19.

1. Preensamble el bougie con el tubo endotraqueal acoplándolo con un conector para fibrobroncoscopía en el conector $15 / 22 \mathrm{~cm}$, y éste a un filtro de partículas de alta eficiencia conectado de manera primaria al ventilador mecánico.

2. El complejo del tubo endotraqueal-bougie-conector-filtro se acopla a la hoja del videolaringoscopio. 
3. Se realiza la videolaringoscopía procurando obtener la mejor visión del anillo glótico en el menor tiempo posible.

4. Se desliza el bougie a través de las cuerdas vocales aproximadamente más de 7 a $10 \mathrm{~cm}$ de las cuerdas vocales.

5. Se desliza el tubo endotraqueal a través de las cuerdas vocales, una vez avanzado el globo retentivo se infla mediante manómetro de presión.

6. Antes de iniciar la ventilación se retira e bougie a través del conector de fibrobroncoscopía de manera lenta y cuidadosa $^{(16)}$.

7. Se ocluye el conector de fibrobroncoscopía y se inicia la ventilación mecánica.

La preocupación de algunos autores con los videolaringoscopios con pantalla acoplada a la pala es la posible contaminación de ésta; sin embargo, se recomienda la colocación de una bolsa plástica que aísle la pantalla de la hoja desechable. Esto no afectaría la visualización y reducirá la posible contaminación de la pantalla con la hoja desechable ${ }^{(17)}$.

1. De igual manera se pide realizar el montaje tubo-bougieconector de filtro y ventilador mecánico.

2. Realice la mejor técnica posible de videolaringo e introduzca el complejo bougie, tubo a través de las cuerdas vocales.

3. Infle el globo con el manómetro a presión adecuada.

4. Retire de manera lenta y gentil el bougie.

5. Ocluya el conector del fibrobroncoscopio e inicio de la ventilación a presión positiva.

La técnica es igual que la técnica anterior para el caso del uso de videolaringoscopio con pantalla desacoplada a la pala, sólo se recomienda tener la pantalla lo más aislada posible. Se recomienda cubrir el cable de la pala del videolaringo y la pantalla con una película o material plástico transparente que evite que el equipo se pueda contaminar con alguna secreción del paciente. Recomendamos el uso de un bougie en todos los escenarios para garantizar el éxito de la intubación ${ }^{(18)}$.

No se recomienda el uso del laringoscopio estándar, debido al riesgo de contacto con el paciente. No se reco-
Tabla 4: Puntaje MACOCHA: escala de Mallampati III o IV,

síndrome de apnea (obstructiva), limitación cervical, apertura

bucal (opening) $<3 \mathrm{~cm}$, coma, hipoxemia, no anestesiólogo.

Factores

Puntuación

Relacionados con el paciente

Mallampati III o IV

SAOS (síndrome de la apnea obstructiva del sueño)

Reducción de la movilidad de la columna cervical

Limitación de la apertura (opening) bucal $<3 \mathrm{~cm}$

Factores relacionados con la patología

Coma

Hipoxemia severa (saturación $\mathrm{O}_{2}<80 \%$ )

Factores relacionados con el operador

No anestesiólogo

Total

5

2

1

1

1

1

1

12

Cálculo de puntaje MACOCHA (originalmente de 25). Puntuación de 0 a 12: el límite que indica una intubación traqueal difícil es $\geq 3$.

American Thoracic Society. Copyright 2017 American Thoracic Society (18)

mienda el uso de máscaras laríngeas ante el elevado riesgo de generación y fuga de aerosoles. Es necesario identificar el riesgo de aspiración y la dificultad en la intubación y en las técnicas de rescate.

Para determinar la presencia de vía aérea difícil se recomienda el puntaje de MACOCHA y la secuencia de palpación laríngea que permita identificar la membrana cricotiroidea (Tabla 4) ${ }^{(20)}$.

\section{CONCLUSIONES}

El manejo de la vía aérea y de la intubación orotraqueal son procedimientos de muy alto riesgo que pueden propagar la transmisión del virus SARS-Cov-2 entre el personal de salud. Por este motivo deben de extremarse las medidas de prevención al realizar estos procesos. Es prioritario que cada institución, con base en la evidencia científica y los recursos disponibles, desarrolle protocolos y procesos adecuados a sus necesidades y se adhiera a las buenas prácticas de calidad y seguridad en la atención.

\section{REFERENCIAS}

1. Bogoch II, Watts A, Thomas-Bachli A, Huber C, Kraemer MU, Khan K Potential for global spread of a novel coronavirus from China. J Travel Med. 2020;27:1-3.

2. Wu P, Hao X, Lau EHY, Wong JY, Leung KSM, Wu JT, Cowling BJ, Leung GM. Real-time tentative assessment of the epidemiological characteristics of novel coronavirus infections in Wuhan, China. Euro Surveill. 2020;25:pii=2000044. doi: 10.2807/1560-7917.

3. Huang C, Wang Y, Li X, Ren L, Zhao J, Hu Y, et al. Clinical features of patients infected with 2019 novel coronavirus in Wuhan, China. Lancet. 2020;497-506.
4. Leung CC, Joynt GM, Gomersall CD, et al. Comparison of high- flow nasal cannula versus oxygen face mask for environmental bacterial contamination in critically ill pneumonia patients: a randomized controlled crossover trial. J Hosp Infect. 2019;84-87.

5. Wong J, Goh QY, Tan Z, Lie SA, Tay YC, Ng SY, et al. Preparing for a COVID-19, pandemic: a review of operating room outbreak response measures in a large tertiary hospital in Singapore. Can J Anaesth. 2020. doi: 10.1007/s12630-020-01620-9.

6. Zhao S, Ling K, Yan H, Zhong L, Peng X, Yao S, et al. Anesthetic management of patients with suspected or confirmed 2019 novel 
coronavirus infection during emergency procedures. J Cardiothorac Vasc Anesth. 2020;1053-0770.

7. Public Health. Enfermedad por el nuevo coronavirus (COVID19). [Videofile]. 2 marzo 2020. Disponible en: https://youtu.be/kKz_vNGsNhc, https://www.youtube.com/watch?v=kKz_vNGsNhc\&feature=youtu.be.

8. Novel Coronavirus Pneumonia Emergency Response Epidemiology Team. The epidemiological characteristics of an outbreak of 2019 novel coronavirus diseases (COVID-19) in China. Chin J Epidemiol. 2020;4:145-151.

9. World Health Organization. Clinical management of severe acute respiratory infections when novel coronavirus is suspected: What to do and what not to do. WHO;2020. Available in: https://www.who.int/csr/ disease/coronavirus_infections/InterimGuidance_ClinicalManagement_ NovelCoronavirus_11Feb13u.pdf.

10. Janz DR, Semler MW, Joffe AM, Casey JD, Lentz RJ, deBoisblanc $\mathrm{BP}$, et al. A multicenter randomized trial of a checklist for endotracheal intubation of critically ill adults. Chest. 2018;153:816-824.

11. Cook TM. Strategies for the prevention of airway complications. A narrative review. Anaesthesia. 2018;73:93-111.

12. Higgs A, McGrath BA, Goddard C, Rangasami J, Suntharalingam G, Gale R, et al. Guidelines for the management of tracheal intubation in critically ill adults. Br J Anaesth. 2018;120:323-352.

13. Gómez-Ríos MA, Gaitini L, Matter I, Somri M. Guidelines andalgorithms for managing the difficult airway. Rev Esp Anestesiol Reanim. 2018;65:41-48.

14. Apfelbaum JL, Hagberg CA, Caplan RA, Blitt CD, Connis RT, Nickinovich DG, et al. Practice guidelines for management of the difficult airway: an updated report by the American Society of Anesthesiologists Task Force on Management of the Difficult Airway. Anesthesiology. 2013;118:251e70.

15. Peng PWH, Ho PL, Hota SS. Outbreak of a new coronavirus: what anaesthetists should know. Br J Anaesth. 2020. doi: S00070912(20)30098-2.

16. Wax RS, Christian MD. Practical recommendations for critical care and anesthesiology teams caring for novel coronavirus (2019-nCoV) patients. Can J Anesth. 2020; doi: org/10.1007/s12630-020-01591-x.

17. Zuo MZ, Huang YG, Ma WH, Xue ZG, Zhang JQ, Gong YH, et al. Expert recommendations for tracheal intubation in critically ill patients with novel coronavirus disease 2019. Chin Med Sci J. 2020. doi: $10.24920 / 003724$.

18. Lewis SR, Butler AR, Parker J, Cook TM, Schofield-Robinson OJ, Smith AF. Videolaryngoscopy versus direct laryngoscopy for adult patients requiring tracheal intubation: a Cochrane Systematic Review. Br J Anaesth. 2017;119:369-383.

19. Quintard H, L'Her E, Pottecher J, Adnet F, Constantin JM, De Jong A, et al. Experts' guidelines of intubation and extubation of the ICU patient of French Society of Anaesthesia and Intensive Care Medicine (SFAR) and French-speaking Intensive Care Society (SRLF): In collaboration with the pediatric Association of French-speaking Anaesthetists and Intensivists (ADARPEF), French-speaking Group of Intensive Care and Paediatric emergencies (GFRUP) and Intensive Care physiotherapy society (SKR). Ann Intensive Care. 2019;9:13. doi: 10.1186/s13613019-0483-1. 